\title{
Generalized strict feasibility and solvability for generalized vector equilibrium problem with set-valued map in reflexive Banach spaces
}

\author{
Gang Wang ${ }^{1 *}$ and Hai-tao Che $^{2}$
}

\footnotetext{
* Correspondence: wgglj1977@163. com

${ }^{1}$ School of Management Science, Qufu Normal University, Shandong Rizhao, 276826, China

Full list of author information is available at the end of the article
}

\begin{abstract}
In this article, the strict feasibility and solvability of generalized vector equilibrium problem with set-valued mapping in reflexive Banach spaces are considered. By introducing two generalized strict feasibility concepts for generalized vector equilibrium problem, we establish some sufficient conditions to guarantee that the solution set of the generalized vector equilibrium problem is nonempty and bounded provided that it is generalized strictly feasible.

MSC: 49K30; 90C29.

Keywords: generalized vector equilibrium problem, nonemptiness and boundedness, generalized strict feasibility
\end{abstract}

\section{Introduction}

Let $X$ be a real reflexive Banach space and $U$ be a metric space, and $K \subseteq X, D \subseteq U$ be two nonempty and closed sets. Let $T: K \rightarrow 2^{D}$ be a nonempty-compact-valued mapping, i.e., $T(x)$ is a nonempty compact subset for any $x \in K$, and upper semicontinuous on $K$. Let $F: D \times K \times K \rightarrow 2^{Y}$ be a set-valued map, where $Y$ is a real normed space with an ordered cone $C$, that is, a proper, closed, and convex cone such that int $C \neq \varnothing$.

The weak generalized vector equilibrium problem [1-4], abbreviated by WGVEP, is to find $\bar{x} \in K$ and $\bar{u} \in T(\bar{x})$ such that

$$
\text { (WGVEP) } \quad F(\bar{u}, \bar{x}, y) \nsubseteq-\text { int } C, \quad \forall y \in K \text {. }
$$

For the WGVEP, its dual problem is to find $\bar{x} \in K$ such that

$$
\text { (DWGVEP) } \quad F(v, y, \bar{x}) \nsubseteq \text { int } C, \quad \forall y \in K, v \in T(y) \text {. }
$$

We denote the solution set of the WGVEP and the solution set of the DWGVEP by $W S_{K}$ and $W S_{K}^{D}$, respectively.

The strong generalized vector equilibrium problem [5,6], abbreviated by SGVEP, is to find $\bar{x} \in K$ and $\bar{u} \in T(\bar{x})$ such that

$$
\text { (SGVEP) } \quad F(\bar{u}, \bar{x}, y) \bigcap-\text { int } C=\emptyset, \quad \forall y \in K \text {. }
$$

\section{空

(C) 2012 Wang and Che; licensee Springer. This is an Open Access article distributed under the terms of the Creative Commons Attribution License (http://creativecommons.org/licenses/by/2.0), which permits unrestricted use, distribution, and reproduction in any medium, provided the original work is properly cited. 
For the SGVEP, its dual problem is to find $\bar{x} \in K$ such that

$$
\text { (DSGVEP) } \quad F(v, y, \bar{x}) \bigcap \text { int } C=\emptyset, \forall y \in K, v \in T(y) .
$$

Similarly, we denote the solution set of the SGVEP and the solution set of the DWGVEP by $S S_{K}$ and $S S_{K}^{D}$, respectively. Obviously,

$$
S S_{K} \subseteq W S_{K}
$$

The generalized vector equilibrium problem finds applications in economics, finance, image reconstruction, ecology, transportation, network, and elasticity in [7]. In particular, when $T(x)$ is singleton, i.e., $T$ is a single-valued map, then the WGVEP collapse to the problem considered in [1-4], and the SGVEP collapse to the problem considered in $[5,6]$. In this case, based on the coercivity assumption, the existence of solution for the generalized vector equilibrium problem are deeply discussed, see [1-13]. Recently, by virtue of the recession method, Ansari established some necessary and/or sufficient conditions for the nonemptiness and boundedness of the solution set for the SGVEP [5]. Later, Farajzadeh and Amini established some sufficient conditions for the compactness and convexity of the solution set of the SGVEP without the requirement of the lower semi-continuity of the map $y \rightarrow F(x, y)$ [6]. Lin derived some existence results for the generalized vector quasi-equilibrium problem under pseudomonotonicity and $u$-hemicontinuity/l-hemicontinuity [11]. Al-Homidan proposed existence results for generalized vector quasi-equilibrium problems by establishing some new fixed point theorems and maximal element theorems [12,13]. Since the WGVEP and the SGVEP are the generalizations of the generalized vector equilibrium problem when $T$ is a single-valued map, it is natural to ask whether the existence of the solution and duality for the WGVEP and the SGVEP can be derived for that $T(x)$ is multivalued, which constitutes the motivation of this article.

Generally, the existence of solution for the classical vector equilibrium problem is established under the strict feasibility condition which was originally used in scalar variational inequality and vector variational inequality [14-17]. This technique can be extended to the scalar equilibrium problem [18]. On the other way, $\mathrm{Hu}$ and Fang extended the concept of strict feasibility to the classical vector equilibrium problem and established the nonemptyness and boundedness of the solution set of the $C$-pseudomonotone vector equilibrium problem if it is strictly feasible in the strong sense [19]. Motivated the study above, in this article, we first investigate the relations between solution set of the WGVEP (SGVEP) and solution set of the WDGVEP (SDGVEP) under the weakly (strongly) $C$-pseudomonotone condition. Furthermore, by introducing two new concepts for strictly feasible in the generalized sense to match the solvability of the WGVEP and the SGVEP, we establish some sufficient conditions to guarantee the nonemptyness and boundedness of the solution set for the generalized vector equilibrium problem if it is generalized strictly feasible. Our results generalize and extend some results of $[18,19]$ in some sense.

\section{Notations and preliminaries}

In this section, we recall some notations and preliminary results needed in the following sections. Let $X, Y, K, D, C, T, F$ be same as in Section 1 .

Definition 2.1 Let $K \subseteq X$ be a nonempty, closed, and convex set. 
(i) The mapping $F: K \rightarrow 2^{Y}$ is said to be $C$-convex if

$$
\alpha F(x)+(1-\alpha) F(y) \subseteq F(\alpha x+(1-\alpha) y)+C, \forall x, y \in K, \alpha \in[0,1]
$$

(ii) The mapping $F: K \rightarrow 2^{Y}$ is said to be C-lower semicontinuous if the set $\{x \in K \mid F$ $(x)-a \nsubseteq$ int $C\}$ is closed on $K$ for any $a \in Y$. F is said to be weakly C-lower semicontinuous if $F$ is $C$-lower semicontinuous with respect to the weak topology of $X$. The map $F$ is said to be weakly lower semicontinuous on $K$ if it is weakly lower semicontinuous on K.

(iii) The mapping $F: D \times K \times K \rightarrow 2^{Y}$ is said to be: weakly C-pseudomonotone if for all $x, y \in K, u \in T(x), v \in T(y)$,

$\exists u \in T(x)$ such that $F(u, x, y) \nsubseteq$ - int $C \Rightarrow \forall v \in T(y)$ such that $F(v, y, x) \nsubseteq$ int $C$, or equivalently,

$\exists v \in T(y)$ such that $F(v, y, x) \nsubseteq$ int $C \Rightarrow \forall u \in T(x)$ such that $F(u, x, y) \subseteq$ - int $C$.

The mapping $F: D \times K \times K \rightarrow 2^{Y}$ is said to be: strongly $C$-pseudomonotone if for all $x, y \in K, u \in T(x), v \in T(y)$,

$\exists u \in T(x)$ such that $F(u, x, y) \cap$-int $C=\varnothing \Rightarrow \forall v \in T(y)$ such that $F(v, y, x) \cap$ int $C=$ $\varnothing$, or equivalently,

$\exists v \in T(y)$ such that $F(v, y, x) \cap$ int $C \neq \varnothing \Rightarrow \forall u \in T(x)$ such that $F(u, x, y) \cap$-int $C \neq$ $\varnothing$,

(iv) The asymptotic cone $K_{\infty}$ and barrier cone $\operatorname{barr}(K)$ of $K$ are, respectively, defined by

$$
K_{\infty}=\left\{d \in X \mid \exists t_{k} \rightarrow+\infty, \exists x_{k} \in K \text { with } \frac{x_{k}}{t_{k}} \rightarrow d\right\}
$$

and

$$
\operatorname{barr}(K)=\left\{x^{*} \in X^{*} \mid \sup _{x \in K}\left\langle x^{*}, x\right\rangle<+\infty\right\},
$$

where $X^{*}$ denotes the dual space of $X$ and $\rightarrow$ stands for the weak convergence.

Remark 2.1 (i) Definition 2.1 is a set-valued generalization of C-lower semicontinuity in $[8]$

(ii) If the map is strongly C-pseudomonotone, then it is weakly C-pseudomonotone. How-ever, the converse result is not true.

Example 2.1 Let $X=R, K=[1,+\infty), Y=R^{2}, C=R_{+}^{2}, T(x)=\{0,-1\}$.

Let $F: D \times K \times K \rightarrow 2^{Y}$ be defined by

$$
F(u, x, y)= \begin{cases}\langle u,[(y-x),|y-x|]\rangle & \forall x, y \in[1,+\infty), u \in T(x) \\ {[1,2]} & \forall x, y \in[1,+\infty), u \in T(x),\end{cases}
$$

$\forall x \in[1,+\infty)$, take $u=0 \in T(x)$, we have

$$
F(u, x, y)=\{0\} \times[1,2] \nsubseteq-\text { int } C .
$$


Its dual problem is:

$$
F(v, y, x)= \begin{cases}\langle v,[(x-y),|x-y|]\rangle & \forall x, y \in[1,+\infty), v \in T(y) \\ {[1,2]} & \forall x, y \in[1,+\infty), v \in T(y) .\end{cases}
$$

$\forall x \in[1,+\infty)$, if $v=0 \in T(y)$, we have

$$
F(v, y, x)=\{0\} \times[1,2] \nsubseteq \text { int } C ;
$$

if $v=-1 \in T(y)$, it holds

$$
F(v, y, x)=[-|x-y|,-(x-y)] \times[1,2] \nsubseteq \text { int } C .
$$

It is easy to see

$$
F(v, y, x) \nsubseteq \text { int } C, \forall y \in[1,+\infty), v \in T(y) \text {. }
$$

Hence $F$ is weakly $C$-pseudomonotone. However, $F$ is not strongly $C$-pseudomonotone.

The asymptotic cone $K_{\infty}$ has the following useful properties.

Lemma 2.1 [20]Let $K \subset X$ be nonempty and closed. Then the following conclusions hold:

(i) $K_{\infty}$ is closed cone;

(ii) If $K$ is convex, then $K_{\infty}=\{d \in X \mid K+d \subset K\}=\{d \in X \mid x+t d \in K, \forall t>0\}$, where $x \in K$ is arbitrary point;

(iii) If $K$ is convex cone, then $K_{\infty}=K$.

Definition 2.2 The GVEP is said to be

(i) generalized strictly feasible in the weak sense if $F_{w}{ }^{+} \neq \varnothing$, where

$$
F_{w}{ }^{+}=\left\{x \in K \mid F(u, x, x+y) \bigcap \text { int } C \neq \emptyset, \forall y \in K_{\infty} \backslash\{0\}, u \in T(x)\right\} ;
$$

(ii) generalized strictly feasible in the strong sense if ${F_{s}^{+}}^{+} \neq \varnothing$, where

$$
F_{s}^{+}=\left\{x \in K \mid F(u, x, x+y) \subseteq \text { int } C, \forall y \in K_{\infty} \backslash\{0\}, u \in T(x)\right\} .
$$

Obviously, both $F_{w}{ }^{+},{F_{s}}^{+}$are equivalent to the $F_{s}^{+}$[19], when $F$ is a single-valued map.

The following example is to explain that Definition 2.2 is applicable.

Example 2.2 Let $X=R, K=[1,+\infty), Y=R, C=R_{+}, T(x)=\{1\}$.

Let $F_{1}: D \times K \times K \rightarrow 2^{Y}$ be defined by

$$
F_{1}(u, x, y)=\langle u,[-(y-x), y-x]\rangle, \forall x, y \in[1,+\infty), u \in T(x) .
$$


It is verified that $K_{\infty}=[0,+\infty)$. For any $x \in[1,+\infty)$ and $t \in K_{\infty} \mid\{0\}$, one has

$$
F_{1}(u, x, x+t)=\langle 1,[-t, t]\rangle=[-t, t] \bigcap \operatorname{int} C \neq \emptyset .
$$

So, $F_{w}{ }^{+}=[1,+\infty)$. However, $F_{s}^{+}=\varnothing$.

Let $F_{2}: D \times K \times K \rightarrow 2^{Y}$ be defined by

$$
F_{2}(u, x, y)=\langle u,[(y-x), 2(y-x)]\rangle, \forall x, y \in[1,+\infty), u \in T(x) .
$$

It is verified that $K_{\infty}=[0,+\infty)$. For any $x \in[1,+\infty)$ and $t \in K_{\infty} \backslash\{0\}$, one has

$$
F_{2}(u, x, x+t)=\langle 1,[t, 2 t]\rangle=[t, 2 t] \subseteq \operatorname{int} C .
$$

So, $F_{w}{ }^{+}=F_{s}{ }^{+}=[1,+\infty)$.

Definition 2.3 [21]A set-valued map $F: E \rightarrow 2^{X}$ is said to be KKM mapping if, for each finite set $\Lambda=\left\{x_{1}, \ldots, x_{n}\right\} \subseteq E$, one has co $\Lambda \subseteq \bigcup_{i=1}^{n} F\left(x_{i}\right)$, where co(.) stands for the convex hull.

The main tools for proving our results are the following well-known KKM theorems.

Lemma 2.2 [22] Assume that $X$ is a topological vector space, $E \subseteq X$ is a nonempty convex and $F: E \rightarrow 2^{X}$ is a KKM mapping with closed values. If there is a subset $X_{0}$ contained in a compact convex subset of $E$ such that $\bigcap_{x \in X_{0}} F(x)$ is compact, then $\bigcap_{x \in E}$ $F(x) \neq \varnothing$.

Definition 2.4 [23,24] Let $K$ be a nonempty, closed, and convex subset of a real reflexive Banach space $X$ with its dual $X *$ We say that $K$ is well-positioned iff there exist $x_{0}$ $\in X$ and $g \in X^{*}$ such that

$$
\left\langle g, x-x_{0}\right\rangle \geq\left\|x-x_{0}\right\|, \forall x \in K .
$$

Lemma 2.3 [23,24]Let $K$ be a nonempty, closed, and convex subset of a real reflexive Banach space $X$ with its dual $X^{*}$. Then $K$ is well-positioned if and only if the barrier cone $\operatorname{barr}(K)$ of $K$ has a nonempty interior. Furthermore, if $K$ is well-positioned then there is no sequence $\left\{x_{n}\right\} \subseteq K$ with $\left\|x_{n}\right\| \rightarrow+\infty$ such that origin is a weak limit of $\left\{\frac{x_{n}}{\left\|x_{n}\right\|}\right\}$.

Lemma 2.4 [25] Let $X$ and $Y$ be two metric spaces and $T: X \rightarrow 2^{Y}$ be a nonemptycompact-valued mapping and upper semicontinuous at $x^{*}$. Then, for any sequences $x_{n}$ $\rightarrow x^{*}$ and $u_{n} \in T\left(x_{n}\right)$, there exist a subsequence $\left\{u_{n_{k}}\right\}$ of $\left\{u_{n}\right\}$ and some $u^{*} \in T\left(x^{*}\right)$ such that $u_{n_{k}} \rightarrow u^{*}$.

\section{Solvability of the WGVEP and the SGVEP}

First, we investigate relations between solution set of the WGVEP (SGVEP) and solution set of the DWGVEP (DSGVEP) when $K$ is bounded.

Theorem 3.1 Let $K \subseteq X$ be a nonempty and convex closed bounded set. If $F: D \times K$ $\times K \rightarrow 2^{Y}$ satisfies the followings:

(i) $F(u, x, x) \subseteq C, \forall x \in K, u \in T(x)$;

(ii) the set $\{(u, x), u \in T(x), x \in K: F(u, x, y) \nsubseteq$-int $C\}$ is closed for any $y \in K$;

(iii) $F$ is weakly $C$-pseudomonotone; 
(iv) the set $\{y \in K \mid F(u, x, y) \nsubseteq$ int $C\}$ is closed and $F(u, x,$.$) is C$-convex for any $x$ $\in K, u \in T(x)$.

Then the WGVEP has a nonempty solution set and $x^{*} \in K$ is a solution of the WGVEP if and only if

$$
F\left(v, y, x^{*}\right) \not \subseteq \text { int } C, \forall y \in K, v \in T(y) .
$$

Proof. Set $\Gamma: D \times K \rightarrow 2^{K}$ by

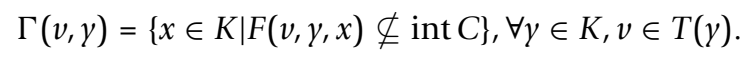

We claim that $\Gamma$ is a KKM map. Suppose on the contrary, it does not hold, then there exists a finite set $\left\{x_{1}, \ldots, x_{n}\right\} \subseteq K$ and $z \in \operatorname{co}\left\{x_{1}, \ldots, x_{n}\right\}$ such that $z \notin \bigcup_{i=1}^{n} \Gamma\left(v, x_{i}\right)$. Thus, there exists $v_{i} \in T\left(x_{i}\right)$ such that $F\left(v_{i}, x_{i}, z\right) \subseteq$ int $C, \forall i=1, \ldots$, $n$. It follows from the weak $C$-pseudomonotonity of $F$ that

$$
F\left(u, z, x_{i}\right) \subseteq-\operatorname{int} C, \forall i=1, \ldots, n .
$$

Taking into account that int $C$ is convex, we obtain

$$
t_{1} F\left(u, z, x_{1}\right)+\ldots+t_{n} F\left(u, z, x_{n}\right) \subset-\operatorname{int} C,
$$

where $z=\sum_{1}^{n} t_{i} x_{i}$ and $\sum_{1}^{n} t_{i}=1, t_{i} \geq 0, i=1,2, \ldots, n$. For the above $t_{i}$, due to the convexity of $F(u, x,$.$) , one has$

$$
t_{1} F\left(u, z, x_{1}\right)+\ldots t_{n} F\left(u, z, x_{n}\right) \subseteq F(u, z, z)+C \subseteq C+C \subseteq C,
$$

which contradicts (3.1). By the condition (iv), we derive that the $\Gamma$ is closed valued. Hence $\Gamma$ is a KKM map. By the KKM Theorem, there exists $x^{*} \in K$ such that $x^{*} \in \cap_{v \in}$ $T(y), y \in K \Gamma(v, y)$. That is, $F\left(v, y, x^{*}\right) \nsubseteq$ int $C, \forall y \in K, v \in T(y)$.

Let us verify $W S_{K}^{D} \subseteq W S_{K}$. Take any $x^{*} \in K$, obviously

$$
F\left(v, y, x^{*}\right) \nsubseteq \text { int } C, \forall y \in K, v \in T(y) .
$$

For every $y \in K$, consider $x_{t}=x^{*}+t\left(y-x^{*}\right), \forall t \in(0,1)$. Clearly, $x_{t} \in K$. The $C$-convexity of $F\left(u, x_{t}.\right)$ implies that

$$
(1-t) F\left(u, x_{t}, x^{*}\right)+t F\left(u, x_{t}, y\right) \subseteq F\left(u, x_{t}, x_{t}\right)+C \subseteq C+C \subseteq C .
$$

Let us show $t F\left(u, x_{b} y\right) \nsubseteq$-int $C$ by contradiction. Suppose on the contrary, then $t F$ $\left(u, x_{b} y\right) \subseteq$-int $C$. For any $p \in t F\left(u, x_{b} y\right)$, it holds

$$
(1-t) F\left(u, x_{t}, x^{*}\right) \subseteq C+p \subseteq C+\operatorname{int} C \subseteq \operatorname{int} C .
$$

So $F\left(u, x_{t} x^{*}\right) \subseteq$ int $C$, which contradicts (3.2). Noting that -int $C$ is convex cone, we deduce

$$
F\left(u, x_{t}, y\right) \nsubseteq \text {-int } C
$$

Letting $t \rightarrow 0$ in (3.3), we obtain by assumption (ii) and Lemma 2.4 that there exists $u^{*} \in T\left(x^{*}\right)$ such that

$$
F\left(u^{*}, x^{*}, y\right) \nsubseteq-\operatorname{int} C, \forall y \in K .
$$


On the other hand, by the weak $C$-pseudomonotonity of $F$, we have $W S_{K} \subseteq W S_{K}^{D}$. Hence, $W S_{K}^{D}=W S_{K}$.

Theorem 3.2 Let $K \subseteq X$ be a nonempty and convex closed bounded set. If $F: D \times K$ $\times K \rightarrow 2^{Y}$ satisfies the followings:

(i) $F(u, x, x) \subseteq C, \forall x \in K, u \in T(x)$;

(ii) the set $\{(u, x), u \in T(x), x \in K \mid F(u, x, y) \cap$-int $C=\varnothing\}$ is closed for all $y \in K$;

(iii) $F$ is strongly $C$-pseudomonotone;

(iv) the set $\{y \in K \mid F(u, x, y) \cap$ int $C=\varnothing\}$ is closed and $F(u, x$, .) is $C$-convex for any $x \in K, u \in T(x)$.

Then the SGVEP has a nonempty solution set and $x^{*} \in K$ is a solution of the SGVEP if and only if

$$
F\left(v, y, x^{*}\right) \bigcap \text { int } C=\emptyset, \forall y \in K, v \in T(y) .
$$

Proof. Set $\Gamma: D \times K \rightarrow 2^{K}$ by

$$
\Gamma(v, y)=\{x \in K \mid F(v, y, x) \bigcap \operatorname{int} C=\emptyset\}, \forall y \in K, v \in T(y) .
$$

Following the similar arguments in the proof of Theorem 3.1, we can obtain the desired result.

In following sequel, we shall present some sufficient conditions for the nonemptiness and boundedness of the solution set of the WGVEP provided that it is strictly feasible in the strong sense.

Theorem 3.3 Let $K \subseteq X$ be a nonempty, closed, convex and well-positioned set. If $F$ : $D \times K \times K \rightarrow 2^{Y}$ satisfies the followings:

(i) $F(u, x, x) \subseteq C, \forall x \in K, u \in T(x)$;

(ii) the set $\{(u, x), u \in T(x), x \in K \mid F(u, x, y) \nsubseteq$-int $C\}$ is closed for any $y \in K$;

(iii) $F$ is weakly $C$-pseudomonotone;

(iv) $F(u, x$, .) is C-convex and weakly lower semicontinuous for $x \in K, u \in T(x)$.

Then the WGVEP has a nonempty bounded solution set whenever it is generalized strictly feasible in the strong sense.

Proof. Suppose that the WGVEP is generalized strictly feasible in the strong sense. Then there exists $x_{0} \in K$ such that $x_{0} \in F_{s}^{+}$, i.e.,

$$
F\left(u, x_{0}, x_{0}+z\right) \subseteq \text { int } C, \forall u \in T\left(x_{0}\right) \text {. }
$$

Set

$$
D=\left\{x \in K \mid F\left(u, x_{0}, x\right) \nsubseteq \text { int } C\right\}, \forall u \in T\left(x_{0}\right) .
$$

By assumptions (i) and (iv), $x_{0} \in D$ and $D$ is weakly closed. We assert that $D$ is bounded. Suppose on the contrary it does not holds, then there exists a sequence $\left\{x_{n}\right\}$ $\subseteq M$ with $\left\|x_{n}\right\| \rightarrow+\infty$ as $n \rightarrow+\infty$. Since $X$ is a reflexive Banach space, without loss of generality, 
we may take a subsequence $\left\{x_{n_{k}}\right\}$ of $\left\{x_{n}\right\}$ such that

$$
\frac{1}{\left\|x_{n_{k}}-x_{0}\right\|} \in(0,1), \lim _{k \rightarrow+\infty} \frac{x_{n_{k}}-x_{0}}{\left\|x_{n_{k}}-x_{0}\right\|}=\lim _{k \rightarrow+\infty} \frac{x_{n_{k}}}{\left\|x_{n_{k}}\right\|} \rightarrow z \in K_{\infty} .
$$

By Lemma 2.3, $z \neq 0$ since $K$ is well-positioned. It follows from $x_{0} \in F_{s}^{+}$that

$$
F\left(u, x_{0}, x_{0}+z\right) \subseteq \operatorname{int} C .
$$

Noting that $F(u, x,$.$) is \mathrm{C}$-convex, we have

$$
\begin{aligned}
& \left(1-\frac{1}{\left\|x_{n_{k}}-x_{0}\right\|}\right) F\left(u, x_{0}, x_{0}\right)+\frac{1}{\left\|x_{n_{k}}-x_{0}\right\|} F\left(u, x_{0}, x_{n_{k}}\right) \subseteq F\left(u, x_{0},\left(1-\frac{1}{\left\|x_{n_{k}}-x_{0}\right\|}\right) x_{0}+\frac{x_{n_{k}}}{\left\|x_{n_{k}}-x_{0}\right\|}\right)+C \\
& \quad=F\left(u, x_{0}, x_{0}+\frac{x_{n_{k}}-x_{0}}{\left\|x_{x_{k}}-x_{0}\right\|}\right)+C .
\end{aligned}
$$

That is,

$$
\frac{1}{\left\|x_{n}-x_{0}\right\|} F\left(u, x_{0}, x_{n_{k}}\right) \subseteq F\left(u, x_{0}, x_{0}+\frac{x_{n_{k}}-x_{0}}{\left\|x_{n_{k}}-x_{0}\right\|}\right)+C .
$$

We claim that $F\left(u, x_{0}, x_{0}+\frac{x_{n_{n}}-x_{0}}{\left\|x_{n_{k}}-x_{0}\right\|}\right) \nsubseteq$ int $C$. Suppose on the contrary, $F\left(u, x_{0}, x_{0}+\frac{x_{n_{h}}-x_{0}}{\left\|x_{n_{k}}-x_{0}\right\|}\right) \subseteq$ inC, we observe

$$
\frac{1}{\left\|x_{n_{k}}-x_{0}\right\|} F\left(u, x_{0}, x_{n_{k}}\right) \subseteq F\left(u, x_{0}, x_{0}+\frac{x_{n_{k}}-x_{0}}{\left\|x_{n_{k}}-x_{0}\right\|}\right)+C \subseteq \text { in } C+C \subseteq \text { int } C,
$$

which contradicts $F\left(u, x_{0}, x_{n_{k}}\right) \nsubseteq$ int $C$. Taking into account the condition (iv), we obtain

$$
F\left(u, x_{0}, x_{0}+z\right) \nsubseteq \text { int } C .
$$

This is a contradiction to (3.4). Thus, $D$ is bounded and it is weakly compact. For each $p \in K$, set

$$
D_{p}=\{x \in D \mid F(v, p, x) \nsubseteq \operatorname{int} C\}, \forall p \in K, v \in T(p) .
$$

Then $D_{p} \neq \varnothing$. Indeed, given $p \in K, v \in T(p)$, set $K_{0}=\operatorname{conv}(D \cup p) \subseteq K$, where conv means the convex hull of a set. Then $K_{0}$ is nonempty, convex, and weakly compact. By Theorem 3.1, there exists $\bar{x} \in K_{0}$ such that

$$
F(v, y, \bar{x}) \nsubseteq \nsubseteq \text { int } C, \forall y \in K_{0}, v \in T(p) .
$$

Then $F\left(u, x_{0}, \bar{x}\right) \nsubseteq$ int $C$ implies $\bar{x} \in D$ and $F(v, p, \bar{x}) \nsubseteq \nsubseteq$ int $C$ implies $\bar{x} \in D_{p}$. We obtain $D_{p} \neq \varnothing$. Obviously, $D_{p}$ is nonempty and weakly compact.

Next we prove that $\left\{D_{p} \mid p \in K\right\}$ has the finite intersection property. For any finite set $\left\{p_{i} \mid i=1,2, \ldots, n\right\} \subseteq K$, let $K_{1}=\operatorname{conv}\left\{D \cup\left\{p_{1}, p_{2}, \ldots, p_{n}\right\}\right\}$. Then $K_{1}$ is weakly compact. By Theorem 3.1, there exists $\hat{x} \in K_{1}$ such that

$$
F(v, y, \hat{x}) \not \nsubseteq \text { int } C, \forall y \in K_{1}, v \in T(p) .
$$

In particular, it holds

$$
F\left(u, x_{0}, \hat{x}\right) \nsubseteq \text { int } C, F\left(v, p_{i}, \hat{x}\right) \nsubseteq \text { int } C, i=1,2, \ldots, n .
$$


This means that $\hat{x} \in \bigcup_{i=1}^{n} D_{p_{i}}$ Thus $\left\{D_{p} \mid p \in K\right\}$ has the finite intersection property. Since $D$ is weakly compact and $D_{p} \subseteq D$ is weakly closed for all $p \in K, v \in T(p)$, It follows that

$$
\bigcap_{p \in K} D_{p} \neq \emptyset .
$$

Let $x^{*} \in \cap_{p \in K} D_{p}$ It follows that

$$
F\left(v, y, x^{*}\right) \nsubseteq \text { int } C, \forall y \in K, v \in T(y) .
$$

By Theorem 3.1, $x^{*}$ is a solution of the WGVEP. As for the boundedness of the solution set of the WGVEP, it follows from Theorem 3.1 that the solution set of the WGVEP is a subset of $D$.

Theorem 3.4 Let $K \subseteq X$ be a nonempty, closed, convex, and well-positioned set. If $F$ : $D \times K \times K \rightarrow 2^{Y}$ satisfies the followings:

(i) $F(u, x, x) \subseteq C, \forall x \in K, u \in T(x)$;

(ii) the set $\{(u, x), u \in T(x), x \in K \mid F(u, x, y) \cap$ - int $C=\varnothing\}$ is closed for all $y \in K$;

(iii) $F$ is strongly $C$-pseudomonotone;

(iv) $F(u, x,$.$) is C$-convex and weakly lower semicontinuous for $x \in K, u \in T(x)$;

(v) $F$ is positively homogeneous with degree $\alpha>0$, i.e., there exists $\alpha>0$ such that

$$
F(u, x, x+t(y-x))=t^{\alpha} F(u, x, y), \forall x, y \in K, u \in T(x), t \in(0,1) .
$$

Then the SGVEP has a nonempty bounded solution set whenever it is generalized strictly feasible in the weak sense.

Proof. Suppose that the SGVEP is generalized strictly feasible in the weak sense. Then there exists $x_{0} \in K$ such that $x_{0} \in F_{w}^{+}$, i.e.,

$$
F\left(u, x_{0}, x_{0}+z\right) \bigcap \text { int } C \neq \emptyset .
$$

Set

$$
D=\left\{x \in K \mid F\left(u, x_{0}, x\right) \bigcap \text { int } C=\emptyset\right\} .
$$

By assumptions (i) and (iv), $x_{0} \in D$ and $D$ is weakly closed. We claim that $D$ is bounded. Suppose on the contrary it does not holds, then there exists a sequence $\left\{x_{n}\right\}$ $\subseteq M$ with $\left\|x_{n}\right\| \rightarrow+\infty$ as $n \rightarrow+\infty$. Since $X$ is a reflexive Banach space, without loss of generality, we may take a subsequence $\left\{x_{n_{k}}\right\}$ of $\left\{x_{n}\right\}$ such that

$$
\frac{1}{\left\|x_{n_{k}}\right\|} \in(0,1), \lim _{n \rightarrow+\infty} \frac{x_{n_{k}}}{\left\|x_{n_{k}}\right\|} \rightarrow z \in K_{\infty} .
$$

By Lemma 2.3, $z \neq 0$ since $K$ is well-positioned. It follows from $x_{0} \in F_{w}+$ that

$$
F\left(u, x_{0}, x_{0}+z\right) \bigcap \text { int } C \neq \emptyset .
$$


Since $x_{n_{k}} \in D$ and $F$ is positively homogenous with degree $\alpha>0$, it holds

$$
F\left(u, x_{0}, x_{0}+\frac{x_{n_{k}}-x_{0}}{\left\|x_{n_{k}}\right\|}\right)=\frac{1}{\left\|x_{n_{k}}\right\|^{\alpha}} F\left(u, x_{0}, x_{n_{k}}\right) \bigcap \text { int } C=\emptyset .
$$

Taking into account the condition (iv), we obtain

$$
F\left(u, x_{0}, x_{0}+z\right) \bigcap \text { int } C=\emptyset .
$$

This is a contradiction to (3.5). Thus, $D$ is bounded and it is weakly compact. Following the similar arguments in the proof of Theorem 3.3, we can prove the Theorem 3.4.

Remark 3.1 Assumption (v) of Theorem 3.4 is not new. Clearly, if $F(x, y)=\langle u, y-x\rangle$, $\forall u \in T(x)$, then $F$ is positively homogeneous with degree $=1$.

Remark 3.2 Since $S S_{K} \subseteq W S_{K}$, conditions for the solution set of the SGVEP to be nonempty and bounded are stronger than the WGVEP. Compared with Theorem 3.3, the condition that $F$ is positively homogeneous in Theorem 3.4 is not dropped for the SGVEP.

The following example shows that the converse of Theorem 3.3 or 3.4 is not true in general.

Example 3.1 Let $X=R, K=R, D=[0,1], Y=R, C=R_{+}^{2}$ and

$$
T(x)= \begin{cases}\{1\}, & \text { if } x>0 \\ \{0,1\}, & \text { if } x=0 .\end{cases}
$$

Let $F: D \times K \times K \rightarrow 2^{Y}$ be defined by

$$
F(u, x, y)= \begin{cases}\left\langle u,\left[\frac{\left(y^{2}-x^{2}\right)}{2},\left(y^{2}-x^{2}\right)\right]\right\rangle & \forall x, y \in K, u \in T(x) \\ \langle u,(y-x)\rangle & \forall x, y \in K .\end{cases}
$$

It is easily to see that $K$ is well-positioned and $F$ satisfies assumptions of Theorems 3.3 and 3.4. It can be verified that the WGVEP and the SGVEP have the same solution set $\{0\}$. On the other hand, it is easy to verify that ${F_{w}}^{+}=F_{s}{ }^{+}=\emptyset$.

For general generalized vector equilibrium problem, the following example shows $W S_{K} \neq \varnothing$, but $S S_{K}=\varnothing$.

Example 3.2 Let $X=R, K=R, D=[-1,1], Y=R, C=R_{+}$and

$$
\begin{gathered}
T(x)=\{-1,1\}, \forall x \in K \\
F(u, x, y)=[-1,1], \forall x, y \in K, u \in T(x) .
\end{gathered}
$$

It is obvious that the WGVEP has solution set $W S_{K}=R$, but solution set of the SGVEP $S S_{K}=\varnothing$.

\section{Acknowledgements}

This research was supported by the Natural Science Foundation of China (Grant Nos.11171180, 71101081 and Tianyuan fund for Mathematics, No. 11126233), and Specialized Research Fund for the doctoral Program of Chinese Higher Education (Grant Nos. 20113705120004, 20113705110002). The authors are in debt to the anonymous referees for their numerous insightful comments and constructive suggestions which help improve the presentation of the article. 


\section{Authors' contributions}

All authors carried out the proof. All authors conceived of the study, and participated in its design and coordination. All authors read and approved the final manuscript.

\section{Competing interests}

The authors declare that they have no competing interests.

Received: 26 November 2011 Accepted: 19 March 2012 Published: 19 March 2012

\section{References}

1. Konnov, IV, Yao, JC: Existence of solutions for generalized vector equilibrium problems. J Math Anal Appl. 233, 328-335 (1999). doi:10.1006/jmaa.1999.6312

2. Ansari, QH, Yao, JC: An existence result for the generalized vector equilibrium problems. Appl Math Lett. 12(8):53-56 (1999). doi:10.1016/50893-9659(99)00121-4

3. Ansari, QH, Konnov, IV, Yao, JC: On generalized vector equilibrium problems. Nonlinear Anal. 47, 543-554 (2001). doi:10.1016/S0362-546X(01)00199-7

4. Ansari, QH, Siddiqi, AH, Wu, SY: Existence and duality of generalized vector equilibrium problems. J Math Anal Appl. 259, 115-126 (2001). doi:10.1006/jmaa.2000.7397

5. Ansari, QH, Flores-Bazán, F: Recession methods for generalized vector equilibrium problems. J Math Anal Appl. 321, 132-146 (2006). doi:10.1016/j.jmaa.2005.07.059

6. Farajzadeh, AP, Harandi, AA: On the generalized vector equilibrium problems. J Math Anal Appl. 344, 999-1004 (2008). doi:10.1016/j.jmaa.2008.02.065

7. Gianness, F: Vector Variational Inequalities and Vector Equilibria. Mathematical Theories, 38 of Nonconvex Optimization and Its Applications. Kluwer, The Nether-lands (2000)

8. Bianchi, M, Hadjisavvas, N, Schaible, S: Vector equilibrium problems with generalized monotone bifunctions. J Optim Theory Appl. 92(3):527-542 (1997). doi:10.1023/A:1022603406244

9. Hadjisavvas, N, Schaible, S: From scalar to vector equilibrium problems in the quasi-monotone case. J Optim Theory Appl. 96, 297-309 (1998). doi:10.1023/A:1022666014055

10. Ansari, QH, Konnov, IV, Yao, JC: Characterizations of solutions for vector equilibrium problems. J Optim Theory Appl. 113(3):435-447 (2002). doi:10.1023/A:1015366419163

11. Lin, LJ, Huang, YJ, Ansari, QH: Some existence results for solutions of generalized vector quasi-equilibrium problems. Math Meth Oper Res. 65, 85-98 (2007). doi:10.1007/s00186-006-0102-4

12. Al-Homidan, S, Ansari, QH: Fixed point theorems on product topological semilattice spaces, generalized abstract economies and systems of generalized vector quasi-equilibrium problems. Taiwanese J Math. 15(1):307-330 (2011)

13. Al-Homidan, S, Ansari, QH, Yao, JC: Collectively fixed point and maximal element theorems in topological semilattice spaces. Applicable Anal. 96(6):865-888 (2011)

14. He, YR, Ng, KF: Strict feasibility of generalized complementarity problems. J Austral Math Soc Ser A. 81(1):15-20 (2006). doi:10.1017/S1446788700014609

15. He, YR, Mao, XZ, Zhou, M: Strict feasibility of variational inequalities in reflexive Banach spaces. Acta Math Sin. 23 , $563-570(2007)$

16. Fang, YP, Huang, NJ: Feasibility and solvability for vector complementarity problems. J Optim Theory Appl. 129(3):373-390 (2006). doi:10.1007/s10957-006-9073-0

17. Fang, YP, Huang, NJ: Feasibility and solvability of vector variational inequalities with moving cones in Banach spaces. Nonlinear Anal. 47, 2024-2034 (2009)

18. Hu, R, Fang, YP: Feasibility-solvability theorem for a generalized system. J Optim Theory Appl. 142(3):493-499 (2009). doi:10.1007/s10957-009-9510-y

19. Hu, R, Fang, YP: Strict feasibility and solvability for vector equilibrium problems in reflexive Banach spaces. Optim Lett. 5, 505-514 (2011). doi:10.1007/s11590-010-0215-9

20. Auslender, A, Teboulle, M: Asymptotic Cones and Functions in Optimization and-Variational Inequalities. Springer, New York (2003)

21. Fan, K: Some properties of sets related to fixed point theorems. Math Ann. 266, 519-537 (1984). doi:10.1007/ BF01458545

22. Tarafdar, E: A fixed point theorem quivalent to the Fan-Knaster-Kuratowski-Mazurkiewcz theorem. J Math Anal Appl. 128, 475-479 (1987). doi:10.1016/0022-247X(87)90198-3

23. Adly, S, Ernst, E, Théra, M: On the closedness of the algebraic difference of closed convex sets. J Math Pures Appl. 82(9):1219-1249 (2003). doi:10.1016/50021-7824(03)00024-2

24. Adly, S, Ernst, E, Théra, M: Well-positioned closed convex sets and well-positioned closed convexfunctions. J Global Optim. 29, 337-351 (2004)

25. Wang, G, Huang, XX, Zhang, J, Chen, GY: Levitin-Polyak weii-posedness of generalized vector equilibrium problems with functional constraints. Acta Math Sci. 30, 1400-1412 (2010)

doi:10.1186/1029-242X-2012-66

Cite this article as: Wang and Che: Generalized strict feasibility and solvability for generalized vector equilibrium problem with set-valued map in reflexive Banach spaces. Journal of Inequalities and Applications 2012 2012:66. 